\title{
Fractional flow reserve successfully predicts ischemic stenoses
}

Reducing myocardial ischemia by revascularization improves a patient's functional status and outcome. Coronary angiography is the standard technique for assessing whether patients should be considered for revascularization. However, "angiography is poor in predicting whether a coronary stenosis induces myocardial ischemia or not," says investigator Pim Tonino, who also explains that "stenting nonischemic lesions is not beneficial, and might even harm patients because of the intrinsic risks associated with this procedure".

In a subanalysis of the FAME study, Tonino and colleagues compared fractional flow reserve (FFR) with angiography for guiding percutaneous coronary intervention (PCI) in 509 patients with multivessel coronary artery disease (CAD). FFR is defined as the ratio between distal coronary pressure and aortic pressure during maximal hyperemia, and provides a functional evaluation of the lesion.

FFR established that even in the most severe stenoses, $20 \%$ of lesions did not induce reversible ischemia. "By using FFR, we can characterize a lesion with a very high sensitivity and specificity," comments Tonino. The angiogram often overestimates a lesion's severity and cannot be relied upon to identify ischemia-producing lesions when assessing stenoses between $50 \%$ and $90 \%$.

Exciting times lie ahead-FAME II was launched in 2010, to compare FFR-guided PCI with medical therapy in patients with stable CAD. FAME III will compare FFRguided PCI with CABG surgery in patients with multivessel disease.

\section{Helene Myrvang}

Original article Tonino, P. A. et al. Angiographic versus functional severity of coronary artery stenoses in the FAME study: fractional flow reserve versus angiography in multivessel evaluation. J. Am. Coll. Cardiol. 55, 2816-2821 (2010) 